\title{
UN LUGAR PARA EL FACTOR HUMANO EN MEDIO DE LA PANDEMIA COVID-19. A MODO DE INTRODUCCIÓN
}

\author{
Carmen Valero-Garcés \\ carmen.valero@uah.es \\ (1) $\mathrm{G} \mathrm{R}^{6} \mathrm{O}$
}

\section{Introduction}

El volumen 8 de FITISPos IJ publicado en la primavera de 2021 viene marcado por la pandemia de la COVID 19 y las transformaciones que ha traído y sus consecuencias. Una pandemia que estalló a comienzos del año 2020 y cuyo fin en el momento de la publicación de estas líneas se augura aún lejano si bien la disponibilidad de vacunas y el proceso de vacunación global haya comenzado.

El mundo está viviendo la mayor crisis económica conocida, calificada por el Fondo Monetario Internacional (FMI 2020) como el Gran Confinamiento y solo comparable con otras dos grandes crisis escritas con mayúsculas- la Gran Depresión (1929) y la Gran Recesión (2007-2014). Una crisis que afecta a todos los aspectos de la vida y que nos ha confinado materialmente en nuestras casas y que ha obligado a los gobiernos a limitar los libres movimientos de las personas tanto a nivel local como nacional o internacional. Hace ya más de un año que vivimos en un período de confinamiento de continuas desescaladas. Es como vivir una especie de coma inducido en el que se seda muy profundamente al paciente para darle la oportunidad de recuperarse al consumir menos energía e ir despertándole poco a poco (Estefanía, 2021, P. 8). Hay recaídas y avances, bajo la creencia de que la situación está controlada, de que se ha aprendido de los errores pasados o de que "esta vez es distinto" porque ahora somos más inteligentes y estamos mejor preparados. Sin embargo, cuesta salir.

Uno de los aspectos que esta crisis ha puesto en evidencia son las debilidades del sector público, la poca protección del ciudadano, la fragilidad de servicios esenciales como el cuidado de la salud, el derecho a la educación, el acceso a un trabajo digno o el disfrute de una vivienda decente. Las limitaciones de movimiento y la falta de acceso a los servicios públicos son dos elementos claves que afectan a la migración. La Agenda 2030 de Naciones Unidas, bajo el lema "Transformar nuestro mundo", garantizaba un apoyo a las personas y países más desfavorecidos al promover un plan de acción en el que las sociedades se debían comprometer para conseguir un futuro sostenible y adecuado para todos. Esta Agenda 2030 se articula en torno a una serie de objetivos interrelacionados entre sí que abordan los desafíos globales a los que nos enfrentamos día a día, como son la pobreza, la desigualdad, el clima, la degradación ambiental, la prosperidad, la paz y la justicia. La COVID-19 irrumpió de forma inesperada a comienzo del 2020 y todo empezó a girar en torno a esta crisis, pasando de lo global a lo local en nuestro entorno, pero con la paradoja de ser más globales que nunca, estar más informados que nunca, tener el mundo a nuestros pies gracias a internet y la tecnología. 
Los extraordinarios avances tecnológicos de las últimas décadas sobre la traducción automática o la inteligencia artificial habían supuesto ya un tremendo avance para la comunicación intralingüística, sobre todo en lenguas mayoritarias o en ámbitos especializados. La COVID-19 disparó el interés y uso de la tecnología y se ha dado un gran salto en la creación de las TIC (Tecnología de la Información y la Comunicación). Tales avances no son, sin embargo, homogéneos y la brecha digital aumenta cuando se trata de lenguas de menor difusión (LMD) o lengua de la migración. Herramientas de uso habitual como Google Translate o DeepL o los múltiples dispositivos móviles de libre acceso en el mercado son prácticamente inexistentes en muchas de las LMD que oímos en nuestras calles, mientras se avanza a un mercado digital único en el que el inglés se erige como lengua franca. Sin embargo, desde una perspectiva más cercana a los objetivos de la Agenda 2030, se trata más bien de otra utopía tecnocrática, una utopía basada en la idea de que todo puede resolverse con la tecnología y con una lengua. La publicación de Tecnología al servicio de la TISP en situaciones de crisis/ Technology at the Service of PSIT in Crisis Situations: Experiences and Perspectives (Valero Garcés, 2018), fruto de la reunión de expertos de diferentes ámbitos con el objetivo de conocer e intercambiar recursos y experiencias sobre la importancia de la TA y herramientas TAO en situaciones de crisis, contiene reflexiones interesantes a tener en cuenta.

Las cuestiones lingüísticas no suelen reconocerse como parte integrante de los movimientos migratorios o de la integración social, si bien los profesionales de la comunicación interlingüística saben que el idioma es un componente necesario para consolidar una sociedad estable (Angelelli, 2015, Foulquié et al., 2018, Valero Garcés, 2018, Wallace y Manzó, 2019). Estos investigadores, y con frecuencia traductores y/o intérpretes profesionales y formadores conocen los desafíos a los que se enfrentan en su trabajo: escasez de tiempo y recursos para la preparación de futuros profesionales, diversidad de lenguas y lugares en los que es necesaria la actuación de intérpretes en LMD, o falta de formación de los bilingües que sirve de intérpretes ad hoc en las instituciones. Saben también de los efectos que esta situación está provocando y el papel que en ello desempeña la cuestión lingüística. Achotegui (2020) en su artículo con el significativo título de "El duelo migratorio por la lengua y la cultura en el síndrome de Ulises", plantea la migración como un cambio vital en el que se viven una serie de duelos, entre los cuales el duelo por la lengua es uno de los más significativos que lleva a muchos inmigrantes a padecer el Síndrome del inmigrante con estrés crónico y múltiple o Síndrome de Ulises.

Los autores que incluyen sus trabajos en el volumen 8 de FITISPos IJ son investigadores, profesionales y formadores de traductores y/o intérpretes que han tenido que enfrentarse a esta pandemia y hacer cambios notables en su forma de enseñar e investigar. Digamos que la respuesta didáctica a esta crisis ha sido casi exclusivamente tecnológica, tratando de adaptar las necesidades a la situación de emergencia que se creó. Y esta misma respuesta tecnológica se ha dado en muchos otros ámbitos de nuestra vida cotidiana. Tal situación ha llegado a provocar en algunos sectores, sobre todo los más desfavorecidos, la sensación de que la dimensión humana de los problemas a los que nos enfrentábamos no se estaba teniendo en cuenta. $\mathrm{Y}$ entre los colectivos más desfavorecidos se hallan aquellos que no tienen acceso a la información porque no conocen la lengua de contacto o no tienen acceso a los canales de distribución de la misma porque hablan lenguas minoritarias y necesitan de intermediarios que les ayuden a comunicarse con los servicios públicos.

La formación de estos futuros intermediarios también se ve mediatizada por la tecnología. En la educación superior hay una inclinación general a dirigir la atención de nuestros alumnos hacia el uso de herramientas TAO, adentrase en la TA y la inteligencia artificial o practicar la localización o posedición. Son menos las voces que abogan por la introducción en los planes de estudio de materias relacionadas con otras capacidades del ser 
humano como la reflexión, la empatía, manejo de estrés, relaciones sociales, colaboración, o mediación.

O’Brien (2020), en la conferencia impartida en enero de 2020, justo antes del confinamiento global, menciona los resultados de un breve estudio que llevó a cabo durante un seminario sobre traducción automática e inteligencia artificial en la Summer School in Advance Translation Technology (SATT) en el verano de 2019. O'Brien preguntó a los participantes en el curso, en su mayoría traductores profesionales, qué consideraban que deberían enseñar a sus estudiantes en el futuro y las respuestas fueron: creación/adaptación de contenido; emprendimiento; especialización en transcreación o localización, por ejemplo, de videojuegos, y trabajo colaborativo.

Massey (2020) llama también la atención sobre esa necesidad de ir más allá de la TA y herramientas TAO en las clases: "I feel that one of my jobs as an educator is to educate people to think differently, is to educate the translators of the future to actually take on an active role of language mediation, consultancy and risk management".

$\mathrm{Y}$ continua diciendo:

(...) I wonder, though, whether the future might not be one closer to an intercultural mediator's role of negotiating between stakeholders who represent different cultures in various modes and media, synchronously and/ or asynchronously. We would then have to trim our teaching programs accordingly. We are training people for the markets and needs of now and the future. How can we feed the current market and anticipate future ones, melding what has been with what might be?

Bernardini et al (2020, p. 304), en un artículo con el significativo título "Language service provision in the 21st century: challenges, opportunities and educational perspectives for translation studies", escrito por autores de cuatro países (Italia, Suiza, Alemania e Irlanda), también apuntan en dicha dirección. Partiendo del hecho de que la demanda de traducción interlingüística e intralingüística y de formas afines de comunicación multilingüe y multimodal va a ir en aumento, consideran que parte de esa demanda podrá satisfacerse con la ayuda de la TA o la IA, e incluso sin ser especialistas se podrán satisfacer parte de esa creciente demanda gracias a estas soluciones de ingeniería. Y concluyen diciendo:

We are living in an age of continuous and fast development of new technologies, where "technology" includes entire workflow architectures. This means that the role of the language professional of the future will have to be different from what it was before: we expect that $\mathrm{s} /$ he becomes an advocate for multilingualism as a globalization tool, and possesses the know-how to manage large-scale commercial, as well as not-forprofit, global initiatives that require translation and transcreation.

Fuera de la disciplina de los Estudios de Traducción, hay también voces que llaman ya también la atención sobre la necesidad de ir más allá de educar en las habilidades tecnológicas. Así, en el informe The Future Skills Report (2019) se identifican tres dimensiones cruciales que deben estar presentes en la educación superior del futuro: competencias relacionadas con el desarrollo del contenido, competencias instrumentales y competencias sociales y organizativas. Concentrarse exclusivamente en la segunda de ellas (competencias instrumentales), como se está haciendo en muchos programas en la enseñanza superior, supone dejar de lado otras competencias que pueden llevar al fracaso a muchos futuros profesionales.

Todas estas consideraciones nos llevaron a dedicar el volumen 8 de FITISPos IJ en este año especial de 2021 a un tema del que no se habla todavía mucho pero que sin duda ocupara cientos de páginas una vez superada la crisis y la fase de recuperación de las secuelas que ha dejado. Así con el título de El factor humano en TISP, el grupo FITISPos une sus fuerzas para contribuir a transformar nuestro mundo en línea con los objetivos de la Agenda 2030 de la 
ONU. Poner los artículos de este volumen junto con las publicaciones y material audiovisual incluido en otra publicación con un título similar: El factor humano en el TISP. Investigación y testimonios de la primavera de 2020 / The Human Factor in TISP. Research and Testimonials of Spring 2020 (Valero Garcés ed. 2020), nos sirven para ofrecer una visión amplia de la compleja realidad que es el ser humano y lo vulnerable que se vuelven ante catástrofes de dimensiones globales.

Los títulos de los artículos que se incluyen en este volumen van en esa dirección. En orden alfabético y en inglés o español, nos ofrecen diversidad de temas y métodos de investigación. Burdeus-Domingo, Gagnon, Pointurier y Yvan Leanza en su artículo "Bridges and barriers in public service interpreting training: instructing non-professional longserving interpreters" dan cuenta de un estudio de investigación-acción que les lleva a diseñar un programa de formación en interpretación en los servicios públicos (ISP) específico para intérpretes ad hoc con experiencia, pero sin formación, ajustado a las necesidades del grupo para terminar con una serie de sugerencias prácticas que permitan superar las dificultades detectadas en su investigación.

Bruno e Iborra Cuéllar en "Un programa de intervención para fomentar la gestión emocional en un grupo de intérpretes para los servicios públicos", presentan el diseño, implantación y resultados de un curso de gestión emocional ad hoc dirigido a interpretes en los servicios públicos. El estudio se llevó a cabo mediante el seguimiento de dos grupos de discusión y de entrevistas. Los autores concluyen que, gracias a la formación, se observó una clara mejoría en la vida de estos intérpretes para los servicios públicos.

Painca en "Re-enacting the past: the translation of performativity in oral history interviews" explora la conexión entre los Estudios de Traducción e historia oral en un caso concreto, el comunismo rumano, para abordar el tema de la transcripción y el poder de la traducción a la hora de dar a conocer la historia.

Jiménez-Andrés, en "Refugee access to information in online and offline environments: results from focus group discussions", llama la atención sobre la creciente importancia de la tecnología para atender las necesidades de información y comunicación con los refugiados e investigan los recursos de acceso a la información en entornos online y offline en comunidades de refugiados de tres países.

Torrella en "La mediación del intérprete como motor hacia la inclusión y la alianza entre naciones", reflexiona sobre un tema complejo como son las relaciones entre la mediación y la interpretación a través del análisis de los principios fundamentales que recogen los códigos éticos y de la observación de la conducta del intérprete mediador en la práctica.

Szymyślik en "La práctica de la traducción literaria y la enseñanza de lenguas en entornos de integración", presenta una propuesta formativa basada en la traducción de textos literarios usando el inglés como lengua vehicular para el fortalecimiento del aprendizaje del español como lengua extranjera en contextos formativos en los que los discentes proceden de contextos minoritarios o de zonas de conflicto y se encuentren en una situación de integración.

Manfredi y Lázaro Gutiérrez en "Interpretación en los servicios públicos en la oferta académica de grado de Argentina", tal y como el titulo indica, analizan la formación de grado en Argentina para concluir que, aunque la Interpretación en los Servicios Públicos no es una asignatura impartida, hay programas que incluyen formación básica que permite a los graduados adquirir algunas de las técnicas esenciales para poder trabajar en los servicios públicos. Concluyen, no obstante, que sería necesario profundizar en la capacitación de los estudiantes en interpretación en los servicios públicos.

De Wilde, van Hest, Rillof, Van Poucke, presentan otra iniciativa formativa puesta en marcha por la Flemish Government Integration Agency, organismo encargado de formar y certificar a los intérpretes de los servicios públicos. Dicha acción va destinada a formar, 
capacitar y evaluar a los voluntarios, algunos de los cuales ya trabajan como intérpretes ad hoc o "asistentes lingüísticos" sin estar certificados.

La sección Documentos de trabajo, Working Papers, incluye los trabajos de jóvenes investigadores basados en sus Trabajos Fin de Master o Tesis Doctorales

Llerandi en "El derecho a la traducción en crisis sanitarias: la COVID-19 en España, 2020", aborda el problema de la comunicación con la población extranjera en España durante el principio de la crisis sanitaria de la COVID-19 en 2020 a través del análisis de materiales disponibles en otras lenguas utilizando como marco conceptual los principios de disponibilidad, acceso, aceptabilidad y adaptabilidad para determinar el tratamiento que recibe la traducción.

Jiménez Garro, en "An English-Spanish glossary for the study of the artificial pancreas in medical translation", tal y como el título indica, trata de un tema más especializado para dar un ejemplo de elaboración de un glosario e insiste en la necesidad de contar con herramientas de este tipo que contengan términos normalizados para producir traducciones adecuadas y facilitar la comunicación entre expertos.

López Blanco, en "Corrección de textos médicos divulgativos traducidos del inglés al español en el ámbito de los servicios públicos de los Estados Unidos", analiza un corpus de textos médicos divulgativos traducidos del inglés al español provenientes de organizaciones públicas de Estados Unidos para concluir que dichos textos no cumplen los estándares de calidad necesarios para ejercer la función con la que se redactaron los textos originales: informar y educar a la población.

Hadji, en "Terminología e interpretación médico-sanitaria en los servicios públicos en el campo de obstetricia y ginecología: propuesta de un glosario médico-sanitario español-árabe”, ofrece un ejemplo más de ampliación de recursos terminológicos médico-sanitarios en españolárabe con la elaboración de un glosario bilingüe de enfermedades, complicaciones y trastornos ginecológico-obstétricos.

María Ruiz, en "Análisis de la redacción de consentimientos informados en inglés y en español", partiendo de la definición de texto híbrido, compara un corpus de consentimientos informados en español y en inglés para ver si cumplen la función de accesibilidad para los pacientes.

En la sección Entrevista/Interview, contamos con las palabras de Aimee Ansari, en la actualidad directora ejecutiva de Translators Without Borders (TWB), pero que lleva varias décadas trabajando en contextos humanitarios y ha colaborado con la ONU y ONG como Oxfam y Save the Children. En la entrevista nos habla de la labor de TWB y de los nuevos retos que la pandemia suma a la ya delicada situación de los más vulnerables, así como de los proyectos y acciones que TWB tiene en marcha. Aimee nos muestra su confianza en la tecnología, el trabajo colaborativo y el voluntariado.

La sección Reseñas/ Book Review incluye comentarios a varios libros o colecciones de artículos recientes de diferentes temas y diferentes campos, dando cuenta del complejo círculo en el que se integra TISP. Los libros comentados son:

El factor humano en Traducción e Interpretación en los Servicios Públicos. Investigación y testimonios de la primavera de 2020, editado por Carmen Valero-Garcés (2020), comentado por Elena Pérez Estevan.

La interpretación en el contexto sanitario: aspectos metodológicos y análisis de interacción del intérprete con el usuario, por Laura Parrilla Gómez (2019), comentado por María de la Presentación Aguilera.

Translatum Nostrum. La traducción y la interpretación en el ámbito especializado, editado por Carla Botella Tejera, Javier Franco Aixelá y Catalina Iliescu Gheorghiu (2020), comentado por Maria Luengo Cabanillas. 
Traducción económica e investigación en España. Estudio bibliométrico, por Daniel Gallego Hernández (2020), comentado por Dario Manglada.

Lengua entre dos fuegos. Intérpretes en la Guerra Civil española (1936-1939) por Jesús Baigorri Jalón (2019), comentado por Yossi Montes

El Rincón del Investigador/ Research Corner incluye varias tesis doctorales recientemente leídas sobre diversos temas, lo que confirma el creciente interés en TISP en la academia. Estas son:

La enseñanza del lenguaje en la atención sanitaria a través de la traducción: aplicaciones didácticas de la traducción en la enseñanza de lengua extranjera de variación especializada / The teaching of language in health care through translation: didactic applications of translation in the teaching of foreign language of specialized variation por Blanca Aparicio Larrán, Universidad de Cádiz, 2020.

Formación y experiencia profesional como diferenciadores en la actuación de intérpretes sanitarios: un estudio de caso desde la sociología de las profesiones / Training and professional experience as differentiators in the performance of health interpreters: a case study from the sociology of professions por Cristina Álvaro Aranda, Universidad de Alcalá, 2020.

Investigación-acción en traducción e inmigración: la utilidad del estudio pretraductológico para el análisis crítico de la libre circulación en España y en el Reino Unido (un estudio de caso) / Action Research, Translation and Immigration: The Usefulness of a Pretranslation Analysis to Critically Analyse EU Freedom Movement Implementation in Spain and in the UK (A Case Study) por Elena Ruiz Cortés, Universidad de Granada, 2020.

Interpretación telefónica: de la teoría a la práctica y su comparación con la interpretación presencial (chino y español) / Telephone interpreting: from theory to practice and its comparison with face-to-face interpreting (Chinese and Spanish) por Jing Li, Universidad de Alcalá, 2020.

Sobre la traducción de documentos médico-legales (inglés-español): retos terminológicos, traductológicos y culturales en documentos pertenecientes a formularios de solicitud para participar en programas de intercambio académico-culturales en los Estados Unidos. Estudios de casos y aplicaciones profesionales / On the translation of medical-legal documents (English-Spanish): terminological, translation and cultural challenges in documents belonging to application forms to participate in academic-cultural exchange programs in the United States. Case studies and professional applications por María Rubio Gragera, Universidad de Málaga, 2020.

El derecho a la interpretación y a la traducción en el proceso penal: la Directiva 2010/64/UE / The right to interpretation and translation in criminal proceedings: Directive 2010/64/EU por Silvia Ponce González, Universidad Católica de Valencia San Vicente Mártir, 2020.

También se hace referencia a dos proyectos relacionadas con la TISP:

MELINCO, un proyecto sobre mediación lingüística para la cooperación al desarrollo, financiado por la Dirección Xeral de Relacións Exteriores e coa Unión Europea, y cuyo objetivo es llevar a cabo un análisis diagnóstico de la situación de la interpretación y la traducción en este ámbito.

INMIGRA3-CM, proyecto subvencionado por la Consejería de Educación e Investigación de la Comunidad de Madrid y por el Fondo Social Europeo, que investiga factores lingüísticos, comunicativos, culturales y sociales del proceso de integración de la población migrante en la Comunidad de Madrid.

Y se mencionan como herramientas novedosas relacionadas con la TIPS una serie de aplicaciones móviles que ponen en evidencia la importancia que la tecnología va adquiriendo 
en la TISP. Se citan CALD Assist app; MiTA app; The AwezaMed COVID-19 app; Migrant Information Centre (MIC) app y Boostlingo Interpreter app.

Confiamos que disfruten leyendo las distintas secciones y aportaciones del vol. 8 de FITISPos IJ y esperamos seguir contando con su colaboración en futuras ediciones. Únicamente cabe añadir que el contenido de los artículos y distintas secciones son responsabilidad de sus autores.

\section{Referencias}

Achotegui, J. (2020). El duelo migratorio por la lengua y la cultura en el síndrome de Ulises. En C. Valero-Garcés (Ed.), El factor humano en el TISP. Investigación y testimonios de la primavera de 2020 / The Human Factor in TISP. Research and Testimonials of Spring 2020. Servicio de Publicaciones de la Universidad. https://www.youtube.com/watch?v=6e5QdK2rRxA\&t=61s.

Angelelli, C. V. (2015). Study on Public Service Translation in Cross-Border Healthcare. Directorate-General for Translation (European Commission). https://doi.org/10.2782/765472

Bernardini, S., Bouillon, P., Ciobanu, D., van Genabith, J., Hansen-Schirra, S., O'Brien, S., Steiner, E. y Teich, E. (2020). Language service provision in the $21^{\text {st }}$ century: Challenges, opportunities and educational perspectives for translation studies. In S. Noorda, P. Scott \& M. Vukasovic (Eds.), Bologna Process Beyond 2020: Fundamental values of the EHEA (pp. 297-303). Bologna, Italy: Bononia University Press. http://bolognaprocess2019.it/wp-content/uploads/2020/07/bologna-process-beyond2020.pdf

Ehlers, Ulf-D. y Kellermann, S.A. (2019). Future Skills. The future of learning and higher education. https://nextskills.files.wordpress.com/2019/05/2019-05-17-report-vs.15.pdf

Estefanía, J. (2021). El Gran Confinamiento. El País. https://elpais.com/ideas/2021-03-05/elgran-confinamiento.html

Foulquié Rubio, A.I., Vargas-Urpi, M. y Fernández-Pérez, M. (eds.). (2018). Panorama de la traducción y la interpretación en los servicios públicos españoles: una década de cambios, retos y oportunidades. Comares.

International Monetary Fund. (2020). World Economic Outlook, April 2020: The Great Lockdown. https://www.imf.org/en/Publications/WEO/Issues/2020/04/14/weo-april2020.

Massey, G. (2020). Language and intercultural mediation. In O'Brian, S.The Future of Translator Training in the MT Era. In Translating Europe Workshop. Machine translation and the human translator. Tampere Universities https://puolukka.uta.fi/ textmine/events/tew-tampere/OBrien_Tampere_Jan2020.pdf

O'Brien, S. (2020). The Future of Translator Training in the MT Era. Translating Europe Workshop: Machine translation and the human translator, Tampere Universities. https://tuni.cloud.panopto.eu/Panopto/Pages/Viewer.aspx?id=dbb39d58-bae7-4e8f947c-acc3005ea516

The Future Skills Report (2019). https://nextskills.files.wordpress.com/2019/05/2019-05-17report-vs.15.pdf

Valero-Garcés, C. (2019). Training public service interpreters and translators: Facing challenges. Revista de Llengua $i$ Dret, Journal of Language and Law, 71, 88-105. https://doi.org/10.2436/rld.i71.2019.3262

Valero-Garcés, C. (Ed.). (2018). Tecnología al servicio de la TISP en situaciones de crisis/ Technology at the Service of PSIT in Crisis Situations: Experiences and Perspectives. Servicio de Publicaciones de la Universidad. 
Valero-Garcés, C. (Ed.). (2020). El factor humano en el TISP. Investigación y testimonios de la primavera de 2020 / The Human Factor in TISP. Research and Testimonials of Spring 2020. Servicio de Publicaciones de la Universidad.

Wallace, M. \& Monzó Nebot, E. (2019). Legal translation and interpreting in public services: Defining key issues, re-examining policies, and locating the public in public service interpreting and translation. Llengua $y$ Dret, 71, 1-13. http://dx.doi.org/10.2436/rld.i71.2019.3311

How to cite this article?/ ¿Cómo citar este artículo?

Valero-Garcés, C. (2021). Un lugar para el factor humano en medio de la pandemia COVID-19. A modo de introducción. FITISPos-International Journal, 8(1), 1-8. https://doi.org/10.37536/FITISPos-IJ.2021.8.1.288 